\title{
Polyestradiol Phosphate
}

National Cancer Institute

\section{Source}

National Cancer Institute. Polyestradiol Phosphate. NCI Thesaurus. Code C1199.

A synthetic long-acting polymer derivative of the female sex hormone estradiol. As the primary, most potent estrogen hormone produced by the ovaries, estradiol binds to and activates specific nuclear receptors. Estradiol exhibits mild anabolic and metabolic properties, and increases blood coagulability. Administration of polyestradiol phosphate leads to the marked decrease of testosterone levels and may exhibit a bone masspreserving effect in patients with prostatic cancer. ( $\mathrm{NCl04)}$ 